Article

\title{
Genome-Wide Discovery of InDel Markers in Sesame (Sesamum indicum L.) Using ddRADSeq
}

\author{
Sibel Kizil ${ }^{1}$, Merve Basak ${ }^{1}$, Birgul Guden ${ }^{1}$, Hilal Sule Tosun ${ }^{2}$, Bulent Uzun ${ }^{1}$ and Engin Yol ${ }^{1, *}$ \\ 1 Department of Field Crops, Faculty of Agriculture, Akdeniz University, TR-07058 Antalya, Turkey; \\ sibl.kzl@gmail.com (S.K.); basak_1129@hotmail.com (M.B.); birgulguden@akdeniz.edu.tr (B.G.); \\ bulentuzun@akdeniz.edu.tr (B.U.) \\ 2 Department of Plant Protection, Faculty of Agriculture, Akdeniz University, TR-07058 Antalya, Turkey; \\ hilaltosun@akdeniz.edu.tr \\ * Correspondence: enginyol@akdeniz.edu.tr; Tel.: +90-242-224-44-00 (ext. 2520); Fax: +90-242-227-45-64
}

Received: 3 September 2020; Accepted: 22 September 2020; Published: 24 September 2020

\begin{abstract}
The development and validation of different types of molecular markers is crucial to conducting marker-assisted sesame breeding. Insertion-deletion (InDel) markers are highly polymorphic and suitable for low-cost gel-based genotyping. From this perspective, this study aimed to discover and develop InDel markers through bioinformatic analysis of double digest restriction site-associated DNA sequencing (ddRADSeq) data from 95 accessions belonging to the Mediterranean sesame core collection. Bioinformatic analysis indicated the presence of $7477 \mathrm{InDel}$ positions genome wide. Deletions accounted for $61 \%$ of the InDels and short deletions (1-2 bp) were the most abundant type $(94.9 \%)$. On average, InDels of at least $2 \mathrm{bp}$ in length had a frequency of $2.99 \mathrm{InDels} / \mathrm{Mb}$. The 86 InDel sites having length $\geq 8$ bp were detected in genome-wide analysis. These regions can be used for the development of InDel markers considering low-cost genotyping with agarose gels. In order to validate these InDels, a total of 38 InDel regions were selected and primers were successfully amplified. About $13 \%$ of these InDels were in the coding sequences (CDSs) and in the $3^{\prime}$ and $5^{\prime}$ - untranslated regions (UTRs). Furthermore, the efficiencies of these 16 InDel markers were assessed on 32 sesame accessions. The polymorphic information content (PIC) of these 16 markers ranged from 0.06 to 0.62 (average: 0.33 ). These results demonstrated the success of InDel identification and marker development for sesame with the use of ddRADSeq data. These agarose-resolvable InDel markers are expected to be useful for sesame breeders.
\end{abstract}

Keywords: genetic diversity; insertion; deletion; marker; oil crop; sesame

\section{Introduction}

Sesame (Sesamum indicum L.) is an oilseed plant in the family Pedaliaceae that has been cultivated for thousands of years. Sesame has been called "the queen of oil seeds" [1] because of its high levels of nutritional oils and proteins [2]. The oil content of most genotypes ranges from 35\% to $60 \%$ [3], and the highest reported value is $62.7 \%$ [4]. Sesame seed oil has five major fatty acids: oleic acid (C18:1), linoleic acid (C18:2), stearic acid (C18:0), palmitic acid (C16:0), and arachidic acid (C20:0) [5]. Oleic acid and linoleic acids are the predominant fatty acids [6]. In addition, sesame oil contains several unique antioxidant lignans (sesamin and sesamolin), which may reduce the risk of atherosclerosis, cardiovascular disease, and coronary heart disease [7]. These polyphenols also provide resistance to oxidative deterioration [8] and are therefore highly important for the oil industry. Sesame plants grow well in tropical and subtropical climates, can tolerate low soil moisture, require low labor input, and can be grown in pure or mixed stands with diverse crops [9]. Despite these advantages, sesame yield is very low due to the persistence of wild-type traits-nonsynchronous flowering, capsule shattering 
in harvest [10], susceptibility to phyllody disease [11], indeterminate growth [12], late maturation, and low environmental adaptability $[13,14]$. The paucity of genetic diversity in sesame species, in addition to the limited amount of basic research, breeding studies, and international cooperation, have hindered efforts to improve agronomically important traits in sesame species.

DNA markers are highly reliable tools that can provide more rapid and accurate characterization of plants than traditional methods [15]. Researchers have used DNA markers in studies of genomic mapping, biodiversity, and gene tagging. Although sesame is an economically important crop, its improvement by use of DNA markers has lagged behind other major oil crops because it is mainly grown in developing countries [16]. Useful molecular markers, including random amplified polymorphic DNA (RAPD) [17], amplified fragment length polymorphism (AFLP) [18,19], simple sequence repeat (SSR) [16,20-22], sequence-related amplified polymorphism (SRAP) [23], and insertion-deletion (InDel) [24,25] markers, have been developed and widely used in genetic diversity studies. A few mapping and marker-assisted selection studies have also been conducted with the use of AFLP markers for the closed capsule mutant trait [26]; RAPD markers for corolla color [27]; and SSR markers for determinate growth habit [12], male-sterile gene [28], and oil and protein content [2] to improve the efficiency of sesame breeding programs. A large number of single nucleotide polymorphisms (SNPs) have also been identified with the advent of next-generation sequencing technology and have been used for the exploitation of genetic diversity $[29,30]$, the construction of high-density linkage mapping [31-33], and the identification of candidate genes for the improvement of sesame production [34-37].

Corresponding regions of genes and genomes in different plants can have different sequence lengths because of insertions or deletions [38]. These mutations are called InDels, and can be formed by insertion of transposable elements, unequal crossover events between similar repeat copies, or slippage in simple sequence replication [39], and may manifest as loss of function or a non-sense mutation [40]. InDels and SNPs are the most abundant and widely distributed sources of variability in plant genomes [41]. They are highly suitable for mapping, genome-wide association analysis, and other genetic studies. However, InDels are preferable to SNPs in marker-assisted breeding programs because InDel polymorphisms can be visualized with more readily designed primers, basic PCR systems, and agarose gel electrophoresis [42]. There is also evidence of greater polymorphism of InDel markers than SSR markers in sesame [25]. Although previous researchers have used InDel markers in studies of many different crops [43-45], only a few studies have examined the use of these markers in sesame $[24,25,46]$. Consequently, we attempted to develop InDel markers with the use of double digest restriction site-associated DNA sequencing (ddRADSeq) data from 95 sesame accessions compared with a reference genome sequence. The selected markers were also validated on sesame germplasm to evaluate their efficiency.

\section{Results}

We performed quality filtering and then generated $349.86 \mathrm{M}$ raw sequence reads by sequencing 95 sesame accessions using the Illumina HiSeq platform. Among these accessions, the mean number of reads was 3.68 M and the guanine-cytosine (GC) content was 38\% [30]. We processed these filtered data using bioinformatic analysis and identified 7477 InDel sites (Table 1).

Deletions accounted for $61 \%$ of these InDel positions. Their sizes ranged from 1 to $14 \mathrm{bp}$, and $94.9 \%$ had sizes of 1 to $2 \mathrm{bp}$. Single-nucleotide variation was the most common type, followed by bi-nucleotide insertions, and these two types accounted for more than $93 \%$ of the total insertions. Among all InDels, $97.5 \%$ were less than $5 \mathrm{bp}, 2.2 \%$ were between 5 to $10 \mathrm{bp}$, and $0.4 \%$ were more than $10 \mathrm{bp}$ long. Single nucleotide length InDels may arise from read or alignment errors, therefore we separately assessed the statistics for each InDel of which the length was greater than a single nucleotide (Table 2). 
Table 1. The number of insertions-deletions (InDels) identified with double digest restriction site-associated DNA sequencing (ddRADSeq) analysis of 95 sesame accessions.

\begin{tabular}{|c|c|c|c|}
\hline InDel Type & $\begin{array}{c}\text { Indel Size } \\
\text { (bp) }\end{array}$ & Number & $\begin{array}{c}\text { Frequency } \\
(\%)\end{array}$ \\
\hline \multirow{14}{*}{ Insertion } & 1 & 2573 & 88.12 \\
\hline & 2 & 143 & 4.90 \\
\hline & 3 & 77 & 2.64 \\
\hline & 4 & 44 & 1.51 \\
\hline & 5 & 24 & 0.82 \\
\hline & 6 & 13 & 0.45 \\
\hline & 7 & 13 & 0.45 \\
\hline & 8 & 6 & 0.21 \\
\hline & 9 & 9 & 0.31 \\
\hline & 10 & 6 & 0.21 \\
\hline & 11 & 5 & 0.17 \\
\hline & 12 & 6 & 0.21 \\
\hline & 13 & 1 & 0.03 \\
\hline & Total & 2920 & \\
\hline \multirow{15}{*}{ Deletion } & 1 & 4119 & 90.39 \\
\hline & 2 & 204 & 4.48 \\
\hline & 3 & 72 & 1.58 \\
\hline & 4 & 56 & 1.23 \\
\hline & 5 & 33 & 0.72 \\
\hline & 6 & 10 & 0.22 \\
\hline & 7 & 10 & 0.22 \\
\hline & 8 & 9 & 0.20 \\
\hline & 9 & 17 & 0.37 \\
\hline & 10 & 12 & 0.26 \\
\hline & 11 & 5 & 0.11 \\
\hline & 12 & 4 & 0.09 \\
\hline & 13 & 2 & 0.04 \\
\hline & 14 & 4 & 0.09 \\
\hline & Total & 4557 & \\
\hline
\end{tabular}

Table 2. Distribution of insertions-deletions (size $\geq 2 \mathrm{bp}$ ) in the genome.

\begin{tabular}{ccccc}
\hline Chromosome & Number of InDels & Number of Deletions & Number of Insertions & $\begin{array}{c}\text { Frequency } \\
(\text { InDels/Mb) }\end{array}$ \\
\hline Chr1 & 75 & 41 & 34 & 3.70 \\
Chr2 & 54 & 32 & 22 & 2.93 \\
Chr3 & 106 & 61 & 45 & 4.10 \\
Chr4 & 52 & 29 & 23 & 2.53 \\
Chr5 & 40 & 23 & 17 & 2.41 \\
Chr6 & 63 & 32 & 31 & 2.43 \\
Chr7 & 54 & 30 & 24 & 3.22 \\
Chr8 & 81 & 51 & 30 & 3.09 \\
Chr9 & 72 & 40 & 32 & 3.15 \\
Chr10 & 52 & 27 & 25 & 2.67 \\
Chr11 & 39 & 20 & 19 & 2.77 \\
Chr12 & 61 & 31 & 30 & 3.74 \\
Chr13 & 36 & 21 & 15 & 2.19 \\
\hline Total & 785 & 438 & 347 & 38.93 \\
\hline
\end{tabular}

The frequency of InDels that were at least 2 bp long varied among the chromosomes, with the greatest number in chromosome 3 and the smallest number in chromosome 13 (Table 2). Separate analysis of insertions and deletions of this size indicated that chromosome 3 also had the 
greatest numbers of deletions and insertions, and chromosome 13 had the smallest numbers of deletions and insertions. The frequency of InDels of this size varied among chromosomes, and ranged from $2.19 \mathrm{InDels} / \mathrm{Mb}$ (chromosome 13) to $4.10 \mathrm{InDels} / \mathrm{Mb}$ (chromosome 3). We examined InDels of $8 \mathrm{bp}$ and longer for development of InDel markers, based on consideration of their genomic distribution and low-cost genotyping with agarose gels (Table 3).

Table 3. Distribution of insertions-deletions of length $\geq 8 \mathrm{bp}$ in the genome.

\begin{tabular}{ccc}
\hline Chromosome & Number of Deletions & Number of Insertions \\
\hline Chr1 & 5 & 5 \\
Chr2 & 4 & 1 \\
Chr3 & 2 & 6 \\
Chr4 & 3 & 2 \\
Chr5 & 3 & 0 \\
Chr6 & 2 & 1 \\
Chr7 & 3 & 2 \\
Chr8 & 8 & 2 \\
Chr9 & 4 & 5 \\
Chr10 & 0 & 2 \\
Chr11 & 9 & 1 \\
Chr12 & 5 & 3 \\
Chr13 & 5 & 3 \\
\hline Total & 53 & 33 \\
\hline
\end{tabular}

There were 86 InDel sites with a length $\geq 8$ bp found in the sesame genome (Table 3 ) and more than half of these were deletions. Chromosome 11 had the highest number of deletions (9) and chromosome 3 had the most insertions (6). We detected no $\geq 8$-bp deletions in chromosome 10 and no $\geq 8$-bp insertions in chromosome 5 (Table 3). The chromosomal position, sequence, and size information for insertions and deletions are shown in Tables 4 and 5. The longest insertion (13 bp) was in chromosome 9 (physical position: 4042652) and the longest deletions (14 bp) were in chromosome 1 (physical position: 12141886), chromosome 5 (physical position: 12064933), and chromosome 11 (physical positions: 9853924 and 11733409). Identified InDels of length $\geq 8$ bp were analyzed in Integrated Genome Browser (IGB) software to display the regions in their appropriate genomic positions (Figures S1 and S2).

Table 4. Information about insertions of length $\geq 8$ bp identified in this study.

\begin{tabular}{cccc}
\hline Chromosome & Physical Position & Sequence & Size (bp) \\
\hline Chr1 & 1924302 & AAAAAACAGA & 10 \\
Chr1 & 8602437 & TAGTTGAGTAA & 11 \\
Chr1 & 10171409 & CTTTTGTTTGC & 11 \\
Chr1 & 15365977 & ATAACCCT & 8 \\
Chr1 & 15931209 & AAGCATCTGC & 10 \\
Chr2 & 8434594 & TCACTTGCTC & 10 \\
Chr3 & 3933997 & AAAGATCAT & 9 \\
Chr3 & 5681885 & ATAACTTT & 8 \\
Chr3 & 5758231 & AATTGTCTG & 9 \\
Chr3 & 13078175 & TGGATTGAT & 9 \\
Chr3 & 24847064 & CTATCTTGTCTG & 12 \\
Chr3 & 25255054 & GTCAGGCG & 8 \\
Chr4 & 3501848 & AACAGCAAG & 9 \\
Chr4 & 12047194 & TCATAACAATAA & 12 \\
Chr6 & 25170199 & TTAGGATATA & 10 \\
Chr7 & 2567633 & CGAGTTTAG & 9 \\
Chr7 & 11218635 & CGCGCCATGG & 10 \\
Chr8 & 17465130 & GTAGGTAATGGC & 12 \\
\hline
\end{tabular}


Table 4. Cont.

\begin{tabular}{cccc}
\hline Chromosome & Physical Position & Sequence & Size (bp) \\
\hline Chr8 & 22375397 & ATGCAGGTATT & 11 \\
Chr9 & 83648 & TCCATTCTG & 9 \\
Chr9 & 2878824 & TCCCAATTTCG & 11 \\
Chr9 & 4042652 & GATCCAGACCTGA & 13 \\
Chr9 & 7344455 & AACCTAACTTA & 11 \\
Chr9 & 17977272 & ATCTGATTACGT & 12 \\
Chr10 & 1129764 & ATTGTTTTACTA & 12 \\
Chr10 & 16879947 & CAATTGACA & 9 \\
Chr11 & 12599287 & GTTATTACGTGT & 12 \\
Chr12 & 7851788 & AAATCCATG & 9 \\
Chr12 & 12737569 & AAATCTGT & 8 \\
Chr12 & 14130703 & TCTGGGAC & 8 \\
Chr13 & 14413322 & TTATTTTCTC & 10 \\
Chr13 & 14462216 & TGACTAGA & 8 \\
Chr13 & 14465088 & CCTGCTTCT & 9 \\
\hline
\end{tabular}

Table 5. Information about deletions of length $\geq 8 \mathrm{bp}$ identified in this study.

\begin{tabular}{|c|c|c|c|}
\hline Chromosome & Physical Position & Sequence & Size (bp) \\
\hline Chr1 & 12141886 & ATACATAAATATAT & 14 \\
\hline Chr1 & 10684379 & GCGGTCATA & 9 \\
\hline Chr1 & 12499067 & TCATATGG & 8 \\
\hline Chr1 & 18076193 & TTCAACGCA & 9 \\
\hline Chr1 & 19878537 & ATTTTTTATG & 10 \\
\hline Chr2 & 11956920 & CACTTAAAT & 9 \\
\hline Chr2 & 16991276 & ATCCACGTG & 9 \\
\hline Chr2 & 18254320 & GAGTGAGGTTG & 11 \\
\hline Chr2 & 13530605 & CTATTCTAGA & 10 \\
\hline Chr3 & 3159274 & TTCTTCAGC & 9 \\
\hline Chr3 & 16817209 & CCGGTTTTGG & 10 \\
\hline Chr4 & 805760 & TTTTCGGCCC & 10 \\
\hline Chr4 & 10090797 & CACGAAAGTGAA & 12 \\
\hline Chr4 & 16553051 & GTCACCTTTACTG & 13 \\
\hline Chr5 & 4151098 & GAAGATGCAT & 10 \\
\hline Chr5 & 12064933 & TATATGTCCAAGAA & 14 \\
\hline Chr5 & 15770216 & AACCTGAA & 8 \\
\hline Chr6 & 3765637 & ACTTGAGT & 8 \\
\hline Chr6 & 15493394 & GTTCTTGGGTT & 11 \\
\hline Chr7 & 9663535 & TACAGTGA & 8 \\
\hline Chr7 & 13849040 & AGGAGGAAT & 9 \\
\hline Chr7 & 14376520 & ATTCAGGGC & 9 \\
\hline Chr8 & 204960 & AATTATTCTGA & 11 \\
\hline Chr8 & 2232178 & GTATGATTAGG & 11 \\
\hline Chr8 & 12091726 & CAATGGCTA & 9 \\
\hline Chr8 & 12863799 & AATAACACATAA & 12 \\
\hline Chr8 & 17895254 & АСССАААСТ & 9 \\
\hline Chr8 & 23088746 & CGTATGTAAA & 10 \\
\hline Chr8 & 24437543 & CAAAAGCTG & 9 \\
\hline Chr8 & 25510231 & ATATTGCC & 8 \\
\hline Chr9 & 1286207 & AGGCTTAAC & 9 \\
\hline Chr9 & 17162035 & GATGGGTGAG & 10 \\
\hline Chr9 & 22192324 & AATCCACAT & 9 \\
\hline Chr9 & 22693423 & CCGATTCCGTCA & 12 \\
\hline
\end{tabular}


Table 5. Cont.

\begin{tabular}{cccc}
\hline Chromosome & Physical Position & Sequence & Size (bp) \\
\hline Chr11 & 1650384 & TGCATCCCA & 9 \\
Chr11 & 2176613 & GTGATAAGTG & 10 \\
Chr11 & 8335212 & CAGGTTCG & 8 \\
Chr11 & 9853924 & AATCATACGATGAG & 14 \\
Chr11 & 11435807 & GTGCAGAGTA & 10 \\
Chr11 & 13066884 & GACCCTGA & 8 \\
Chr11 & 13584022 & TTATCAAAT & 9 \\
Chr11 & 13826811 & TAAATTTCA & 9 \\
Chr12 & 7621962 & TGCACTAAAT & 10 \\
Chr12 & 11308275 & AAGAAATTT & 9 \\
Chr12 & 12221515 & GCACGACT & 8 \\
Chr12 & 12487265 & AGACTAAC & 8 \\
Chr12 & 12936814 & AATAACTTAG & 10 \\
Chr13 & 8231260 & ACGTCTTGTAGG & 12 \\
Chr13 & 10333430 & AATTATTGATC & 11 \\
Chr13 & 10529779 & TAACAAGCAGTAA & 13 \\
Chr13 & 12075963 & CACCATCAC & 9 \\
Chr13 & 13896532 & TGTATCATAA & 10 \\
\hline
\end{tabular}

A total of 38 InDel regions with lengths $\geq 8$ bp were selected and primers were successfully designed with the primer3 package. These 38 InDels were distributed throughout the 13 chromosomes. Chromosomes 1 and 8 had the greatest number of InDel markers (6), followed by chromosome 9 (5). The clean amplicons were generated with these primers (Figure S3). Analysis of InDel genomic positions indicated that $86.84 \%$ of them were in intergenic regions, $7.90 \%$ were in $5^{\prime}$ untranslated regions (UTRs), $2.63 \%$ were in $3^{\prime}$ UTRs, and $2.63 \%$ were in coding sequences (CDS) (Table 6).

A genetic diversity analysis was conducted with 16 InDel markers across 32 randomly selected sesame accessions from the Mediterranean sesame core collection. PCR products were visualized on a Fragment Analyzer ${ }^{\circledR}$ for all the studied loci (Figure S4) and InDel markers showed the expected polymorphisms within the accessions (Table 7). The observed and expected heterozygosities were found in the range of 0 to 0.25 and 0.02 to 0.47 , respectively. The highest expected heterozygosity value was obtained in loci S-D-5-157, S-D-7-143, and S-D-8-223, and the lowest was seen in loci S-D-1-106 and S-I-4-120, with a mean value of 0.34 . The average Shannon diversity index (I) was found to be 0.49 . The polymorphic information content (PIC) values of these 16 markers ranged from 0.06 to 0.62 , with an average of 0.33 . Principal coordinate analysis (PCoA) indicated that the first and second coordinate explained $27.66 \%$ and $14.93 \%$ of the total variation, respectively. The sesame panel was also divided into three groups including accessions from different continents in the PCoA graphic (Figure 1). The UPGMA tree also showed two distinct groups (Figure 2). 
Table 6. The primer sequences of the 38 InDel markers developed and used in this study.

\begin{tabular}{|c|c|c|c|c|c|c|c|c|}
\hline Marker Name & Chromosome & Physical Position & InDel Type & $\begin{array}{c}\text { Indel } \\
\text { Size (bp) }\end{array}$ & Forward Primer $\left(5^{\prime}\right.$ to $\left.3^{\prime}\right)$ & Reverse Primer $\left(5^{\prime}\right.$ to $\left.3^{\prime}\right)$ & Product Length (bp) & Locus Location * \\
\hline S-D-1-106 & chr1 & 10684379 & Deletion & 9 & GATGAATTTAATTGAGTCCAACAA & ATTTTTCTGACTTAGGTGTTTATGC & 180 & UTR_3 \\
\hline S-D-1-121 & chr1 & 12141886 & Deletion & 14 & TTCCAGGTGGAGATCCTGAC & GGAGCGGAATTCTGGACATA & 202 & intergenic region \\
\hline S-D-1-124 & chr1 & 12499067 & Deletion & 8 & TTGACGAATAATTTTTGTTTTCCA & CCTGGTGGAAATGGAGTCAA & 183 & intergenic region \\
\hline S-D-1-198 & chr1 & 19878537 & Deletion & 10 & TGTGCATCTTTGATACATATGAATTTT & TCACACTGCGTTATTGATTTAATTT & 182 & intergenic region \\
\hline S-D-2-135 & chr2 & 13530605 & Deletion & 10 & CAAATTCACATAACCAGCATTGA & GTCCGGGACGTGAAATTGAT & 244 & intergenic region \\
\hline S-D-4-805 & chr4 & 805760 & Deletion & 10 & AGGCAGACCAGGGTTTTACA & GGTTTTAGCTCTAGAGGAAAGAAAACT & 169 & intergenic region \\
\hline S-D-4-100 & chr4 & 10090797 & Deletion & 12 & GAGCAGCAGCACCCATTAAC & GCAGTGGCTCAATTCTGGTT & 231 & intergenic region \\
\hline S-D-4-165 & chr4 & 16553051 & Deletion & 13 & GGGGAAATGATGGAGGGTTA & CAAGTTCAACGTCACCAATTT & 249 & intergenic region \\
\hline S-D-5-157 & chr5 & 15770216 & Deletion & 8 & GCGAAACACAGCCTAAAAGG & TGTTTGGAGCTTCCTCATTTG & 155 & intergenic region \\
\hline S-D-6-154 & chr6 & 15493394 & Deletion & 11 & GTGTGGCCGGAAATCAAT & TGAAAGCAAACCTCAAGAGTG & 234 & UTR_5 \\
\hline S-D-7-138 & chr7 & 13849040 & Deletion & 9 & TTTTACCTGGGGATTTGAAGG & CTAACGAGGTGGTGGGCAAT & 150 & $\operatorname{CDS}$ \\
\hline S-D-7-143 & chr7 & 14376520 & Deletion & 9 & GGATTTAATCGGGGAAGCAT & TCCGATGTTTTCCTTTCGAG & 217 & intergenic region \\
\hline S-D-8-223 & chr8 & 2232178 & Deletion & 11 & TCCTACGGTTGGATGTTGATG & ACGGGTGCGCTAACAACC & 150 & intergenic region \\
\hline S-D-8-120 & chr8 & 12091726 & Deletion & 9 & CAGGCACCTCAAAGGAAGAG & GGGAGGAGTCGTCTGTCGT & 810 & intergenic region \\
\hline S-D-8-178 & chr8 & 17895254 & Deletion & 9 & GTGTGCCCCTAGTTTCGAGT & GTGAGCTGGCGGTGATTATT & 198 & UTR_5 \\
\hline S-D-8-230 & chr8 & 23088746 & Deletion & 10 & AATTGTATTCGAATCAGGTTTGG & CAGCCATATAGTTGGGTGGA & 150 & intergenic region \\
\hline S-D-8-244 & chr8 & 24437543 & Deletion & 9 & TGATTTTGGGATCTTGAACGA & TTGCCTGCTTTATGTGATGC & 153 & intergenic region \\
\hline S-D-8-255 & chr8 & 25510231 & Deletion & 8 & TCAAGCCTTAATCGGAGACC & TTCTGCTCTCACGCGTATTC & 431 & intergenic region \\
\hline S-D-9-128 & chr9 & 1286207 & Deletion & 9 & TGCATAGCAACATAAATGAGGAA & СTCTTATGCATGGCCACCAC & 103 & intergenic region \\
\hline S-D-9-171 & chr9 & 17162035 & Deletion & 10 & CGGAACTTCTCAGTGATAAAGAGC & ТССАССТGTTССАТССТСТС & 353 & intergenic region \\
\hline S-D-12-113 & chr12 & 11308275 & Deletion & 9 & AATTAGCCGCCTTTTTGGTT & TTGTTTTGAAATTGACGGTACG & 374 & intergenic region \\
\hline S-D-12-124 & chr12 & 12487265 & Deletion & 8 & TGCATGCATCTAAACCTTGAA & AATTTCGGCACATTTCAAAAA & 162 & intergenic region \\
\hline S-D-13-823 & chr13 & 8231260 & Deletion & 12 & GCTTCTTATTCACTTAAATGGTGCT & TCGTCACTTTTTCTAAGAGAGCTT & 233 & intergenic region \\
\hline S-D-13-103 & chr13 & 10333430 & Deletion & 11 & TCTCCGGACTGTCTGAAAGG & TGTCTTTGATCCGTTGGTCA & 626 & intergenic region \\
\hline S-I-1-101 & chr1 & 10171409 & Insertion & 11 & GGGGAGGTAATTATTCCGTGA & TATACACGTCCGCAAGAGCA & 152 & intergenic region \\
\hline S-I-1-192 & chr1 & 1924302 & Insertion & 10 & TCTTCATCTGTCACCCCAAA & CTGTTAAGCGCCACTGTTGA & 173 & intergenic region \\
\hline S-I-3-248 & chr3 & 24847064 & Insertion & 12 & TTTTCACCTGTTTCGAGACCT & CTTTGAGCTGGAACGTGGAT & 174 & intergenic region \\
\hline S-I-4-120 & chr4 & 12047194 & Insertion & 12 & TTGTTGGAAGGACTAAAATTGAAA & GGGCAATGTGCACCTTTTA & 304 & intergenic region \\
\hline S-I-6-251 & chr6 & 25170199 & Insertion & 10 & ATTGCATTTGGGCTGGATTA & CССССТCGAAACAACTAATG & 228 & intergenic region \\
\hline S-I-7-112 & chr7 & 11218635 & Insertion & 10 & GTCACССТCAAGGAGATCCA & AAACAGAAAGAAGAGAAAAACCCTTA & 238 & intergenic region \\
\hline S-I-8-174 & chr8 & 17465130 & Insertion & 12 & CTGCAAGCAACAAACCAAAA & TCTTCAAGAGCTCATGGCTACA & 167 & intergenic region \\
\hline S-I-9-179 & chr9 & 17977272 & Insertion & 12 & CATTCCСТTCAAAACCCACA & TGCAACGCTTGCAAGAAAC & 213 & UTR_5 \\
\hline S-I-9-404 & chr9 & 4042652 & Insertion & 13 & CAGCGGATTTGTGCTTGTTA & GACTCTAACTTTACCCAATTCTTTAGG & 161 & intergenic region \\
\hline S-I-9-836 & chr9 & 83648 & Insertion & 9 & ATGGGCCTGTACCGGTATACTA & TTTTTGAGTGAATGACTATGATTACAT & 223 & intergenic region \\
\hline S-I-10-168 & chr10 & 16879947 & Insertion & 9 & TCTATTCTGACATTGACCGGATT & TCACAAAAACAACCAAAGTTGC & 152 & intergenic region \\
\hline S-I-10-112 & chr10 & 1129764 & Insertion & 12 & TGATGGAGTAATTGAAAGTGTACG & CAAAAGCAGAGTTGACCGTATG & 155 & intergenic region \\
\hline S-I-10-125 & chr11 & 12599287 & Insertion & 12 & GGCAAAGAAATGCAGAGGAG & САСТTТСАСССАСССАТСАТ & 210 & intergenic region \\
\hline
\end{tabular}


Table 7. Summary of genetic diversity statistics for 32 sesame accessions.

\begin{tabular}{cccccccc}
\hline Marker/Locus & Na & Ne & $\boldsymbol{I}$ & Ho & He & F & PIC \\
\hline S-D-1-106 & 1.25 & 1.03 & 0.05 & 0.00 & 0.02 & 1.00 & 0.06 \\
S-D-1-121 & 1.75 & 1.55 & 0.45 & 0.00 & 0.31 & 1.00 & 0.32 \\
S-D-4-165 & 1.75 & 1.55 & 0.45 & 0.00 & 0.31 & 1.00 & 0.32 \\
S-D-5-157 & 2.00 & 1.90 & 0.66 & 0.03 & 0.47 & 0.95 & 0.37 \\
S-D-7-143 & 2.00 & 1.89 & 0.66 & 0.03 & 0.47 & 0.94 & 0.35 \\
S-D-8-223 & 2.00 & 1.83 & 0.64 & 0.15 & 0.45 & 0.61 & 0.37 \\
S-D-8-178 & 2.00 & 1.65 & 0.54 & 0.07 & 0.37 & 0.64 & 0.30 \\
S-D-9-128 & 1.75 & 1.58 & 0.47 & 0.05 & 0.32 & 0.84 & 0.62 \\
S-D-12-124 & 2.00 & 1.69 & 0.58 & 0.25 & 0.40 & 0.33 & 0.31 \\
S-I-1-192 & 2.00 & 1.72 & 0.59 & 0.10 & 0.41 & 0.66 & 0.36 \\
S-I-3-248 & 1.75 & 1.70 & 0.51 & 0.01 & 0.36 & 0.96 & 0.37 \\
S-I-4-120 & 1.25 & 1.12 & 0.13 & 0.01 & 0.08 & 0.82 & 0.19 \\
S-I-6-251 & 2.00 & 1.84 & 0.63 & 0.10 & 0.44 & 0.67 & 0.37 \\
S-I-9-179 & 1.75 & 1.44 & 0.40 & 0.09 & 0.26 & 0.51 & 0.25 \\
S-I-10-168 & 2.00 & 1.73 & 0.60 & 0.12 & 0.41 & 0.71 & 0.32 \\
S-I-10-112 & 1.75 & 1.55 & 0.46 & 0.01 & 0.31 & 0.96 & 0.33 \\
\hline Mean & 1.81 & 1.61 & 0.49 & 0.06 & 0.34 & 0.76 & 0.33 \\
\hline
\end{tabular}

* Number of alleles (Na), effective number of alleles (Ne), Shannon diversity index (I), expected heterozygosity (He), observed heterozygosity (Ho), Wright's fixation index (F), polymorphic information content (PIC).

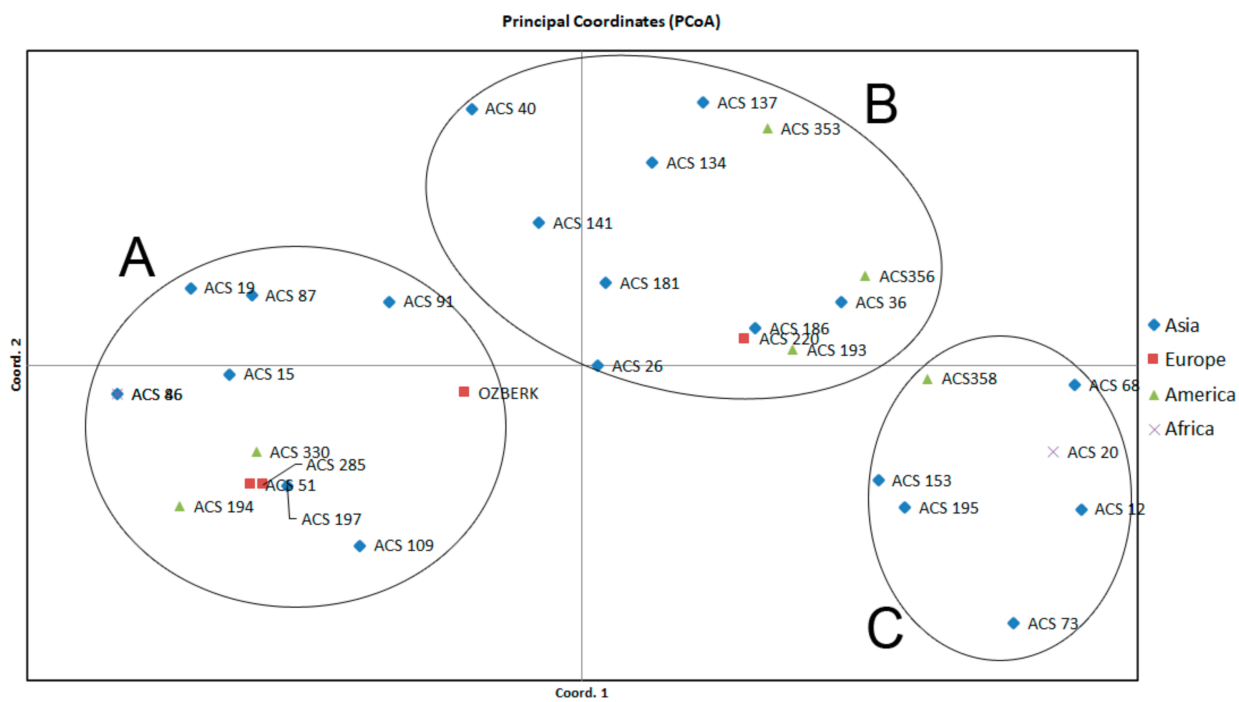

Figure 1. Principal coordinate analysis (PCoA) of the 32 sesame accessions genotyped with $16 \mathrm{nDel}$ markers. (A-C) show the three classes with respect to distribution of genotypes. 


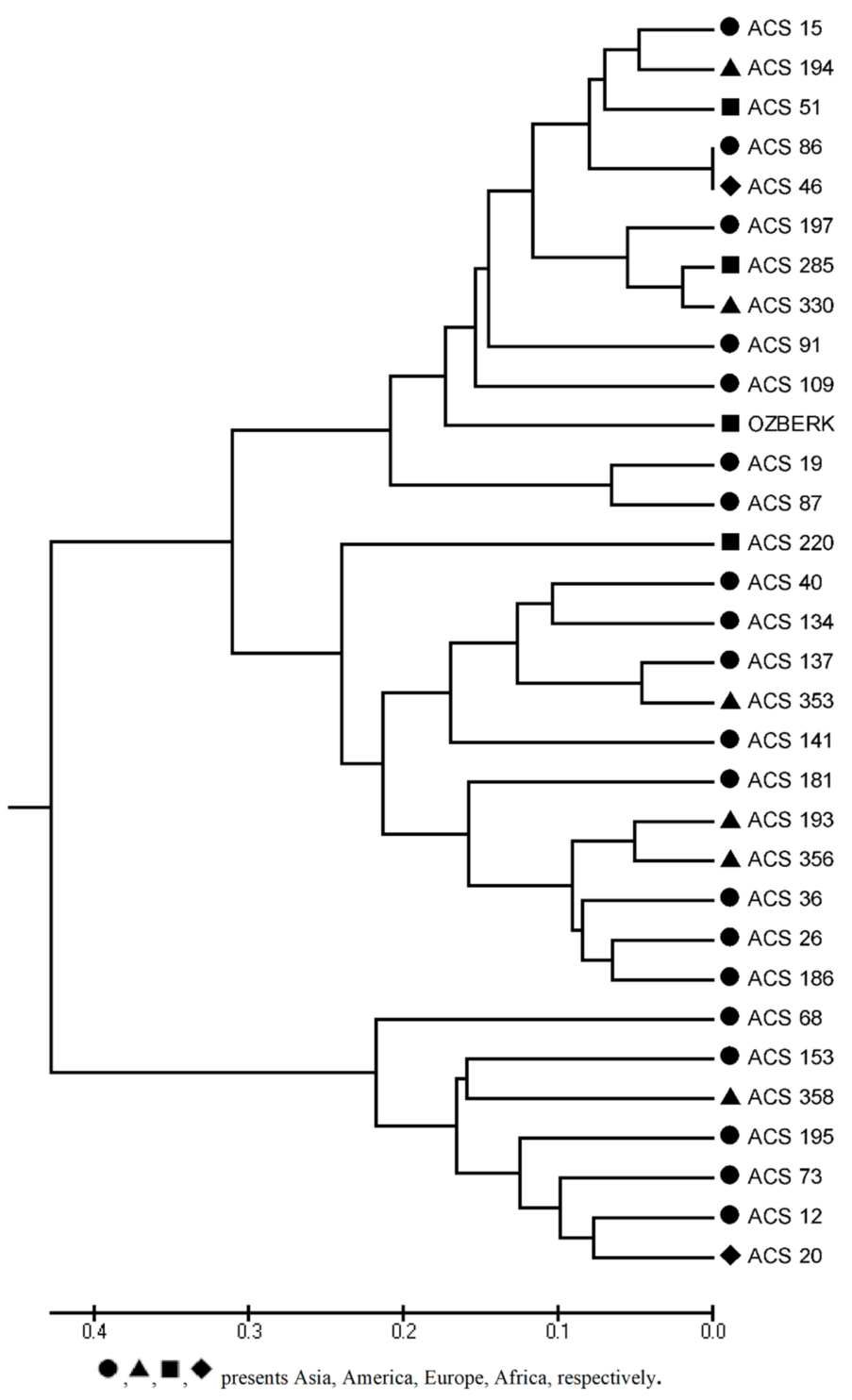

Figure 2. UPGMA dendrogram showing the genetic relationships among the 32 sesame accessions.

\section{Discussion}

ddRADSeq is a cost-effective sequencing protocol that uses two restriction enzymes to reduce genome complexity for SNP discovery and genotyping [47]. We used ddRADSeq to identify 7477 InDel sites, with the ddRADSeq indicating the effectiveness of this protocol to identify InDel regions in the sesame genome. To our knowledge, this is the first successful large-scale development of InDel markers in sesame using ddRADSeq data. The InDels we identified varied among chromosomes, confirming the suitability of this protocol for genome-wide marker development. Therefore, they can be used for the construction of high-density genetic maps, the exploitation of genetic diversity, and the identification of candidate genes. We also presented an optimized procedure for InDel detection using the Galaxy platform (www.usegalaxy.org) that does not require coding processes with stringent bioinformatics settings.

Table 1 showed that a total of 14 InDel classes were detected based on type (insertion vs. deletion) and the number of InDels declined with the increase of InDel size, and the most common type were single-nucleotide InDels. These results are in concordance with previous studies which reported that single-nucleotide InDels were most common in kenaf [48], chickpea [49], and sesame [24]. In contrast, bi-nucleotide InDels were most common in Zea mays [50] and Brassica rapa [44]. Our analysis of InDels 
that were at least $2 \mathrm{bp}$ long indicated the greatest number in chromosome 3 and the smallest number in chromosome 13. This observation is consistent with previous studies of sesame, which reported that the greatest number of simple sequence repeats (SSRs) [16] and SNPs [30] were on chromosome 3. On the other hand, we identified no deletions that were $8 \mathrm{bp}$ or longer in chromosome 10 and no deletions of this length in chromosome 5. This might be a disadvantage of ddRADSeq, because there can be large gaps in the genome coverage after sequencing of a genomic library prepared using this protocol [51]. Our InDel frequency was 1 per $37.74 \mathrm{~kb}$ (7477 InDels in $259.73 \mathrm{Mb}$ ), much higher than the frequency $(1 / 137 \mathrm{~kb})$ obtained by Wei et al. [24], who used transcriptome assembly for InDel detection in sesame. In addition, we found more InDels compared to a study which used restriction site-associated DNA (RAD) sequencing [25]. These differences, therefore, could be a consequence of the sequencing method, the number of genotypes used for genotyping, and bioinformatic parameters for the exploration of variants.

Plant breeders commonly accept agarose gel-based DNA markers more than those markers from newer technologies, such as HRM, KASP, SNP arrays, and PAGE-based SSR, due to the ease of use and the familiarity of the agarose gel system [52]. This led us to develop 38 agarose-resolvable markers and successful amplifications were obtained with bulk DNAs. The lack of PCR failure in individual PCR assays indicates the absence of variation in primer binding sites. In turn, this further shows the power of the ddRADSeq library approach and the InDel filtering pipeline, leading to $100 \%$ success in PCR assays. In addition, we used a single PCR program for amplifying multiple loci, suggesting the potential utility of these markers for multiplex PCR assays. Annotation analysis showed that most of the InDels were in intergenic regions (Table 6), similar to the results of Wei et al. [24], who developed InDel markers from sesame transcriptome data. About $13 \%$ of the developed InDels were in the CDS and the $3^{\prime}$ and $5^{\prime}$ UTRs (Table 6), suggesting that they may be valuable resources for genomics-assisted breeding applications. For example, researchers previously reported an 11-bp deletion in the early flowering 3 gene (ELF3) of chickpea and successfully used this region as an InDel marker [53].

The exploitation of genetic diversity in sesame genetic resources is highly important in order to utilize collections and improve breeding studies. In this study, the effectiveness of the developed markers was assessed on the sesame germplasm, including 32 accessions from four different continents. Genetic diversity analysis showed that the average PIC value of 16 markers was 0.33 , higher than PIC value of the InDel (Wei et al. [54]) and AFLP (Laurentin and Karlovsky [55]) markers used to identify genetic variation in sesame. Previous research also reported a PIC value above 0.50 for SSR markers [56,57] and expressed sequence tag-SSR (EST-SSR) markers [20] in sesame. Botstein et al. [58] categorized the PIC values of markers as highly informative $(\geq 0.5)$, reasonably informative $(0.50$ to $0.25)$, or least informative $(\leq 0.25)$. Our average PIC value $(0.33)$ thus indicates that the markers identified here are reasonably informative and adequate for evaluating relationships among accessions, according to Meszaros et al. [59]. The principal coordinate analysis using 16 InDel markers between 32 sesame accessions revealed three classes, giving no clear pattern with respect to geographical origin. Migration of different accessions by people and/or trade among regions over centuries may explain these results [30]. Previous research also reported that human-related factors may be responsible for the lack of correlation between genetic and geographical distance in other crop plants [60]. Our findings are in agreement with the conclusions of Laurentin and Karlovsky [18], who reported no association between genetic differentiation and accession origin in sesame. Most of the sesame accessions used in PCoA and UPGMA tree analysis based on genetic distance from 16 InDel markers were consistent with a phylogenetic tree analysis conducted with 5292 SNPs [30]. This demonstrates the effectiveness of the new markers, which successfully revealed differences among accessions in the present investigation. In addition, InDel markers showed their ability to reliably discern genetic diversity in sesame collections [25]. 


\section{Materials and Methods}

\subsection{Plant Material and DNA Extraction}

The Mediterranean sesame core collection consists of 103 accessions, and previous studies have characterized their agro-morphological traits [61], oil characteristics [62], and SNP data [30]. The core collection was developed with the principal component score strategy from 345 sesame accessions, considering 12 qualitative and nine quantitative traits [61]. The seeds of each accession in the collection were sown in pots; however, eight of them did not germinate. The remaining 95 accessions in the collection, which were from 21 different regions in Africa, America, Asia, and Europe, were used as a genetic material for ddRADSeq analysis (Table S1). DNA was extracted from young leaves using the CTAB method [63] with minor modifications. The quality and quantity of DNA was checked by electrophoresis on $1 \%$ agarose gels, and the amount was normalized to $100 \mathrm{ng} / \mu \mathrm{L}$ using lambda DNA as a reference.

\section{2. ddRADSeq and InDel Calling}

Before genotyping, random DNA samples were tested with MspI to determine the effectiveness of restriction enzyme digestion. A ddRAD library was prepared using restriction enzymes ( $V s p \mathrm{I}$ and MspI) using a modification of the ddRAD method [47], as described by Basak et al. [30]. A reduced representative genomic library with an insert size of 400-500 bp was subjected to Illumina 150-bp paired-end sequencing. The ddRAD sequencing data of 95 available genotypes (accession number: PRJNA560319) were submitted to the National Center for Biotechnology Information (NCBI) Sequence-Read Archive (SRA) database.

For bioinformatic analysis, the raw data were demultiplexed using Je V1.2 [64], a quality check was conducted for FASTQ Sanger files using fastp [65], and reads with a Phred quality score less than 15 out of 40 and restriction enzyme sequences were trimmed. Each genotype was subsequently aligned with the reference genome sequence "Zhongzhi13 V2.0" [66] using Bowtie2 with default parameters [67] in the Galaxy software framework (www.usegalaxy.org). The resulting BAM files were used in Freebayes (Galaxy Version 1.1.0.46-0) [68], with simple diploid calling and filtering, and coverage values of 20x for variant calling. The resulting variant files were filtered using VCFfilter (Galaxy Version 1.0.0) and SNPs were discarded. The individual .vcf files, which included insertions and deletions, were later merged using VCFgenotypes (Galaxy Version 1.0.0) to form a single data file.

The combined variant file was processed using Microsoft Excel to eliminate duplicated regions and organize the InDels according to their sizes. InDel regions that were at least 8 bp long were checked using the Integrated Genome Browser V9.1.4 (IGB) [69] with each BAM file and the sesame reference genome.

\subsection{Primer Design and PCR Analysis}

Forward and reverse primers from sesame reference genome sequences that flanked the selected InDels were designed using Primer3Plus ([70]; http://www.bioinformatics.nl/cgi-bin/primer3plus/ primer3plus.cgi) to develop genome-wide InDel markers. The length of primer pairs was limited to a minimum of $18 \mathrm{bp}$ and the predicted products ranged from 100 to $900 \mathrm{bp}$. The primer pairs were later controlled for possible duplication of sequences in the genome using IGB software. All markers were named using the format $\mathrm{S}-\mathrm{D}(\mathrm{I})-\mathrm{X}-\mathrm{XXX}$, where " $\mathrm{S}$ " indicates sesame, " $\mathrm{D}$ " and " $\mathrm{I}$ " indicate deletion and insertion, " $X$ " is the chromosome number, and " $X X X$ " is start of the chromosomal position. InDel annotation was based on the sesame reference genome sequence using Generic Feature Format 3 (GFF3) [66].

Primers were checked with random bulk DNAs, and PCR was performed in a $20 \mu \mathrm{L}$ reaction volume with $1 \mu \mathrm{L}$ of $10 \times$ PCR buffer, $2.5 \mathrm{mM} \mathrm{MgCl}_{2}, 0.3 \mu \mathrm{L}$ of dNTP mix $(10 \mathrm{mM}), 0.3 \mu \mathrm{L}$ each of forward and reverse primers $(10 \mu \mathrm{M}), 0.2 \mu \mathrm{L}$ of Taq DNA polymerase $(5 \mathrm{U} / \mu \mathrm{L})$, and $1 \mu \mathrm{L}$ of genomic DNA and Milli-Q water. Thermocycling started at $95^{\circ} \mathrm{C}$ for $2.5 \mathrm{~min}$; followed by 4 cycles of $95^{\circ} \mathrm{C}$ for 
$45 \mathrm{~s}, 50{ }^{\circ} \mathrm{C}$ for $20 \mathrm{~s}$, and $60{ }^{\circ} \mathrm{C}$ for $50 \mathrm{~s} ; 30$ cycles of $92{ }^{\circ} \mathrm{C}$ for $20 \mathrm{~s}, 50{ }^{\circ} \mathrm{C}$ for $20 \mathrm{~s}$, and $60{ }^{\circ} \mathrm{C}$ for $50 \mathrm{~s}$; and a final extension at $60^{\circ} \mathrm{C}$ for $10 \mathrm{~min}$. The PCR products were separated on $2 \%$ agarose gels and visualized by UV light.

To determine their performance, 16 selected InDel markers were used to examine the genetic diversity of the sesame germplasm (32 accessions). PCR conditions were performed as described above. The expected PCR bands were monitored using a Fragment Analyzer ${ }^{\mathrm{TM}}$ (Advanced Analytical Technologies GmbH, Heidelberg, Germany) for accurate sizing. The DNF-900-K0500 reagent kit was used for qualitative analysis of DNA fragments. The solutions, buffers, and gels were prepared according to the manufacturers' instructions. The data were normalized to $1 \mathrm{bp}$ (lowest) and $500 \mathrm{bp}$ (highest), and calibrated to the 1 to $500 \mathrm{bp}$ range DNA ladder. The virtual gel image was assessed using PROSize 2.0 (Advanced Analytical Technologies, AMES, IA, USA).

\subsection{Genetic Diversity Analysis}

Calculations of population genetic parameters, number of alleles $(\mathrm{Na})$, effective number of alleles $(\mathrm{Ne})$, Shannon diversity index (I), expected heterozygosity (He), observed heterozygosity (Ho), Wright's fixation index $(\mathrm{F})$, and principal coordinate analysis (PCoA) were performed using GenAlex V6.5 [71]. The Excel Microsatellite Toolkit [72] was used to measure polymorphism. A phylogenetic tree was constructed based on genetic distance with MEGA 5 [73].

\section{Conclusions}

In this study, a large number of InDels were detected from sequencing of the Mediterranean sesame collection with the use of a ddRADSeq protocol. These results indicated that this technique is an effective approach for the development of genome-wide markers in a short time. Among 86 InDel sites that had lengths of $\geq 8 \mathrm{bp}, 38$ agarose-resolvable markers were successfully amplified and 16 of them were randomly selected to detect polymorphism among 32 sesame accessions. The remaining InDel genomic regions (Tables 4 and 5) identified in this study can therefore be used for the development of InDel markers that might play an important role in different breeding studies, such as the construction of linkage maps, marker-assisted selection (MAS), and gene mapping and selection.

Supplementary Materials: The following are available online at http://www.mdpi.com/2223-7747/9/10/1262/s1, Figure S1: The software Integrated Genome Browser (IGB) shows the deletion in chromosome 1 (position 10.684.379-10.684.387). Gray colors are deleted sequences for each individual in that region. Coordinates indicate the reference genome sequence. Figure S2: The software IGB shows the insertion in chromosome 10 (position $16,879,947-16,879,956)$. Green-black colors are inserted sequences for each individual in that region. Coordinates indicate the reference genome sequence. Figure S3: Amplification of sesame DNAs with use of selected markers (Ladder $100 \mathrm{bp}$ ), Figure S4: Fragment Analyzer ${ }^{\mathrm{TM}}$ shows the sample gel pictures of InDel marker profile for selected sesame accessions with a 1-500 bp ladder. Table S1: List of the sesame accessions in the Mediterranean sesame core collection using ddRADSeq analysis.

Author Contributions: E.Y. designed the research and methodology. S.K., M.B., H.S.T. and B.G. conducted laboratory studies and E.Y. analyzed the sequence data. E.Y. and B.U. wrote the manuscript. All authors have read and agreed to the published version of the manuscript.

Funding: This research received no external funding.

Acknowledgments: The authors are thankful to the Scientific Research Projects Coordination Unit of Akdeniz University for continuous support. We are also grateful to USDA, ARS Plant Genetic Resources Conservation Unit, Griffin, GA, United States for supplying genetic material several times.

Conflicts of Interest: The authors declare no conflict of interest

\section{References}

1. Bedigian, D.; Harlan, J.R. Evidence for cultivation of sesame in the ancient world. Econ. Bot. 1986, 40, 137-154. [CrossRef]

2. Li, C.; Miao, H.; Wei, L.; Zhang, T.; Han, X.; Zhang, H. Association mapping of seed oil and protein content in Sesamum indicum L. using SSR markers. PLoS ONE 2014, 9, e105757. [CrossRef] [PubMed] 
3. Uzun, B.; Arslan, C.; Furat, S. Variation in fatty acid compositions, oil content and oil yield in a germplasm collection of sesame (Sesamum indicum L.). J. Am. Oil Chem. Soc. 2008, 85, 1135-1142. [CrossRef]

4. Arslan, C.; Uzun, B.; Ulger, S.; Cagirgan, M.I. Determination of oil content and fatty acid compositions of sesame mutants suited for intensive management conditions. J. Am. Oil Chem. Soc. 2007, 84, 917-920. [CrossRef]

5. Mondal, N.; Bhat, K.V.; Srivastava, P.S. Variation in fatty acid composition in Indian germplasm of sesame. J. Am. Oil Chem. Soc. 2010, 87, 1263-1269. [CrossRef]

6. Kamal-Eldin, A.; Appelqvist, L.A. Variation in fatty acid composition of the different acyl lipids in seed oils from four Sesamum species. J. Am. Oil Chem. Soc. 1994, 71, 135-139. [CrossRef]

7. Shahidi, F.; Naczk, M. Phenolics in Food and Nutraceuticals; CRC Press: Boca Raton, FL, USA, 2004.

8. Moazzami, A.A.; Kamal-Eldin, A. Sesame seed is a rich source of dietary lignans. J. Am. Oil Chem. Soc. 2006, 83, 719-723. [CrossRef]

9. Ashri, A. Sesame (Sesamum indicum L.). In Genetics Resources, Chromosome Engineering and Crop Improvement; Singh, R.J., Ed.; CRC Press: Boca Raton, FL, USA, 2007; Volume 4, pp. 231-289.

10. Yol, E.; Uzun, B. Inheritance of indehiscent capsule character, heritability and genetic advance analyses in the segregation generations of dehiscent $x$ indehiscent capsules in sesame. Tar. Bil. Der. 2019, 25, 79-85. [CrossRef]

11. Ustun, R.; Yol, E.; Ikten, C.; Catal, M.; Uzun, B. Screening, selection and real-time qPCR validation for phytoplasma resistance in sesame (Sesamum indicum L.). Euphytica 2017, 213, 159. [CrossRef]

12. Uzun, B.; Çagirgan, M.I. Identification of molecular markers linked to determinate growth habit in sesame. Euphytica 2009, 166, 379-384. [CrossRef]

13. Mahajan, R.K.; Bisht, I.S.; Dhillon, B.S. Establishment of a core collection of world sesame (Sesamum indicum L.) germplasm accessions. Sabrao J. Breed. Genet. 2007, 39, 53-64.

14. Furat, S.; Uzun, B. The use of agro-morphological characters for the assessment of genetic diversity in sesame (Sesamum indicum L). Plant Omics 2010, 3, 85-91.

15. Jones, N.; Ougham, H.; Thomas, H.; Pasakinskiene, I. Markers and mapping revisited: Finding your gene. New Phytol. 2009, 183, 935-966. [CrossRef]

16. Dossa, K.; Yu, J.; Liao, B.; Cisse, N.; Zhang, X. Development of highly informative genome-wide single sequence repeat markers for breeding applications in sesame and construction of a web resource: SisatBase. Front. Plant. Sci. 2017, 8, 1470. [CrossRef]

17. Bhat, K.V.; Babrekar, P.P.; Lakhanpaul, S. Study of genetic diversity in Indian and exotic sesame (Sesamum indicum L.) germplasm using random amplified polymorphic DNA (RAPD) markers. Euphytica 1999, 110, 21-33. [CrossRef]

18. Laurentin, H.; Karlovsky, P. Genetic relationship and diversity in a sesame (Sesamum indicum L.) germplasm collection using amplified fragment length polymorphism. BMC Genet. 2006, 7, 10. [CrossRef]

19. Ali, G.M.; Yasumoto, S.; Katsuta, M.S. Assessment of genetic diversity in sesame (Sesamum indicum L.) detected by amplified fragment length polymorphism markers. Electron. J. Biotechnol. 2007, 10, 12-23. [CrossRef]

20. Wei, W.; Qi, X.; Wang, L.; Zhang, Y.; Hua, W.; Li, D.; Lv, H.; Zhang, X. Characterization of the sesame (Sesamum indicum L.) global transcriptome using Illumina paired-end sequencing and development of EST-SSR markers. BMC Genom. 2011, 12, 451. [CrossRef]

21. Zhang, H.; Wei, L.; Miao, H.; Zhang, T.; Wang, C. Development and validation of genic-SSR markers in sesame by RNA-seq. BMC Genom. 2012, 13, 316. [CrossRef]

22. Surapaneni, M.; Yepuri, V.; Vemireddy, L.R.; Ghanta, A.; Siddiq, E.A. Development and characterization of microsatellite markers in Indian sesame (Sesamum indicum L.). Mol. Breed. 2014, 34, 1185-1200. [CrossRef]

23. Ali Al-Somain, B.H.; Migdadi, H.M.; Al-Faifi, S.A.; Salem, S.A.; Abdulmalek, A.M.; Nabil, A.M.; Yahya, A.R. Assessment of genetic diversity of sesame accessions collected from different ecological regions using sequence-related amplified polymorphism markers. 3 Biotech. 2017, 7, 82. [CrossRef] [PubMed]

24. Wei, L.; Miao, H.; Li, C.; Duan, Y.; Niu, J.; Zhang, T.; Zhao, Q.; Zhang, H. Development of SNP and InDel markers via de novo transcriptome assembly in Sesamum indicum L. Mol. Breed. 2014, 34, 2205-2217. [CrossRef] 
25. Wu, K.; Yang, M.; Liu, H.; Tao, Y.; Mei, J.; Zhao, Y. Genetic analysis and molecular characterization of Chinese sesame (Sesamum indicum L.) cultivars using insertion-deletion (InDel) and simple sequence repeat (SSR) markers. BMC Genet. 2014, 15, 35. [CrossRef]

26. Uzun, B.; Lee, D.; Donini, P.; Çağirgan, M.L. Identification of a molecular marker linked to the closed capsule mutant trait in sesame using AFLP. Plant Breed. 2003, 122, 95-97. [CrossRef]

27. Rao, P.V.R.; Prasuna, K.; Anuradha, G.; Srividya, A.; Vemireddy, L.R.; Shankar, V.G.; Sridhar, S.; Jayaprada, M.; Reddy, K.R.; Reddy, N.E.; et al. Molecular mapping of important agro-botanic traits in sesame. Electron. J. Plant. Breed. 2014, 5, 475-488.

28. Liu, H.; Zhou, X.; Wu, K.; Yang, M.; Zhao, Y. Inheritance and molecular mapping of a novel dominant genic male-sterile gene in Sesamum indicum L. Mol. Breed. 2015, 35, 9. [CrossRef]

29. Cui, C.; Mei, H.; Liu, Y.; Zhang, H.; Zheng, Y. Genetic diversity, population structure, and linkage disequilibrium of an association-mapping panel revealed by genome-wide SNP markers in sesame. Plant. Sci. 2017, 8, 1189.

30. Basak, M.; Uzun, B.; Yol, E. Genetic diversity and population structure of the Mediterranean sesame core collection with use of genome-wide SNPs developed by double digest RAD-Seq. PLoS ONE 2019, 14, e0223757. [CrossRef]

31. Zhang, H.; Miao, H.; Wei, L.; Li, C.; Zhao, R.; Wang, C. Genetic analysis and QTL mapping of seed coat color in sesame (Sesamum indicum L.). PLoS ONE 2013, 8, e63898. [CrossRef]

32. Wu, K.; Liu, H.; Yang, M.; Tao, Y.; Ma, H.; Wu, W.; Zuo, Y.; Zhao, Y. High-density genetic map construction and QTLs analysis of grain yield-related traits in sesame (Sesamum indicum L.) based on RAD-Seq techonology. BMC Plant. Biol. 2014, 14, 274. [CrossRef]

33. Mei, H.; Liu, Y.; Du, Z.; Wu, K.; Cui, C.; Jiang, X.; Zhang, H.; Zheng, Y. High-density genetic map construction and gene mapping of basal branching habit and flowers per leaf axil in sesame. Front. Plant. Sci. 2017, 8, 636. [CrossRef] [PubMed]

34. Wei, X.; Liu, K.; Zhang, Y.; Feng, Q.; Wang, L.; Zhao, Y.; Li, D.; Zhao, Q.; Zhu, X.; Zhu, X.; et al. Genetic discovery for oil production and quality in sesame. Nat. Commun. 2015, 6, 8609. [CrossRef] [PubMed]

35. Zhou, R.; Dossa, K.; Li, D.; Yu, J.; You, J.; Wei, X.; Zhang, X. Genome-wide association studies of 39 seed yield-related traits in sesame (Sesamum indicum L.). Int. J. Mol. Sci. 2018, 19, 2794. [CrossRef] [PubMed]

36. Li, D.; Dossa, K.; Zhang, Y.; Wei, X.; Wang, L.; Zhang, Y.; Liu, A.; Zhou, R.; Zhang, X. GWAS uncovers differential genetic bases for drought and salt tolerances in sesame at the germination stage. Genes 2018, 9 , 87. [CrossRef]

37. Du, H.; Zhang, H.; Wei, L.; Li, C.; Duan, Y.; Wang, H. A high-density genetic map constructed using specific length amplified fragment (SLAF) sequencing and QTL mapping of seed-related traits in sesame (Sesamum indicum L.). BMC Plant. Biol. 2019, 19, 588. [CrossRef]

38. Yang, J.; Wang, Y.; Shen, H.; Yang, W. In silico identification and experimental validation of insertion-deletion polymorphisms in tomato genome. DNA Res. 2014, 21, 429-438. [CrossRef]

39. Britten, R.J.; Rowen, L.; Williams, J.; Cameron, R.A. Majority of divergence between closely related DNA samples is due to indels. Proc. Natl. Acad. Sci. USA 2003, 100, 4661-4665. [CrossRef]

40. Rockah-Shmuel, L.; Tóth-Petróczy, A.; Sela, A.; Wurtzel, O.; Sorek, R.; Tawfik, D.S. Correlated occurrence and by pass of frame-shifting insertion-Deletions (InDels) to give functional proteins. PLoS Genet. 2013, 9, e1003882. [CrossRef]

41. Rafalski, A. Applications of single nucleotide polymorphisms in crop genetics. Curr. Opin. Plant. Biol. 2002, 5, 94-100. [CrossRef]

42. Wu, D.; Wu, H.; Wang, C.; Tseng, H.; Hwu, K. Genome-wide InDel marker system for application in rice breeding and mapping studies. Euphytica 2013, 192, 131-143. [CrossRef]

43. Pacurar, D.I.; Pacurar, M.L.; Street, N.; Bussell, J.D.; Pop, T.I.; Gutierrez, L.; Bellini, C. A collection of INDEL markers for mapbased cloning in seven Arabidopsis accessions. J. Exp. Bot. 2012, 63, 2491-2501. [CrossRef] [PubMed]

44. Liu, B.; Wang, Y.; Zhai, W.; Deng, J.; Wang, H.; Cui, Y.; Cheng, F.; Wang, X.; Wu, J. Development of InDel markers for Brassica rapa based on whole-genome re-sequencing. Theor. Appl. Genet. 2013, 126, 231-239. [CrossRef] [PubMed] 
45. Tan, S.; Cheng, J.W.; Zhang, L.; Qin, C.; Nong, D.G.; Li, W.P.; Tang, X.; Wu, Z.M.; Hu, K.L. Construction of an interspecific genetic map based on InDel and SSR for mapping the QTLs affecting the initiation of flower primordia in pepper (Capsicum spp.). PLoS ONE 2015, 10, e0119389. [CrossRef] [PubMed]

46. Wei, X.; Zhu, X.; Yu, J.; Wang, L.; Zhang, Y.; Li, D.; Zhou, R.; Zhang, X. Identification of sesame genomic variations from genome comparison of landrace and variety. Front. Plant. Sci. 2016, 7, 1169. [CrossRef] [PubMed]

47. Peterson, B.K.; Weber, J.N.; Kay, E.H.; Fisher, H.S.; Hoekstra, H.E. Double digest RADseq: An inexpensive method for de novo SNP discovery and genotyping in model and non-model species. PLoS ONE 2012, 7, e37135. [CrossRef] [PubMed]

48. Zhang, L.; Wan, X.; Zhang, L.; Xu, Y.; Xu, J.; Lin, L.; Qi, J.; Zhang, L. Development of InDel markers for identification of a single mendelian locus controlling leaf shape in kenaf (Hibiscus cannabinus). Trop. Plant. Biol. 2019, 12, 78-84. [CrossRef]

49. Jain, A.; Roorkiwal, M.; Kale, S.; Garg, V.; Yadala, R.; Varshney, R.K. InDel markers: An extended marker resource for molecular breeding in chickpea. PLOS ONE 2019, 14, e213999. [CrossRef]

50. Batley, J.; Barker, G.; O'Sullivan, H.; Edwards, K.J.; Edwards, D. Mining for single nucleotide polymorphisms and insertions/deletions in maize expressed sequence tag data. Plant. Physiol. 2003, 132, 84-91. [CrossRef]

51. Shirasawa, K.; Hirakawa, H.; Isobe, S. Analytical workflow of double-digest restriction site-associated DNA sequencing based on empirical and in silico optimization in tomato. DNA Res. 2016, 23, 145-153. [CrossRef]

52. Hu, W.; Zhou, T.; Wang, P.; Wnag, B.; Song, J.; Han, Z.; Chen, L.; Liu, K.; Xing, Y. Development of whole-genome agarose-resolvable LInDel markers in Rice. Rice 2020, 13, 1. [CrossRef]

53. Ridge, S.; Deokar, A.; Lee, R.; Daba, K.; Macknight, R.C.; Weller, J.L.; Tar'an, B. The chickpea early flowering 1 (Efl1) locus is an ortholog of Arabidopsis ELF3. Plant. Physiol. 2017, 175, 802-815. [CrossRef] [PubMed]

54. Wei, L.B.; Miao, H.M.; Xu, F.F.; Kong, J.J.; Zhang, H.Y. Chinese sesame cultivars, DNA fingerprinting, and two-dimensional barcodes using single nucleotide polymorphisms, insertions or deletions, and simple sequence repeat markers. Crop. Sci. 2017, 57, 1941-1947. [CrossRef]

55. Laurentín, H.; Karlovsky, P. AFLP fingerprinting of sesame (Sesamum indicum L.) cultivars: Identification, genetic relationship and comparison of AFLP informativeness Parameters. Genet. Resour Crop. Evol. 2007, 54, 1437-1446. [CrossRef]

56. Park, J.H.; Suresh, S.; Cho, G.T.; Choi, N.G.; Baek, H.J.; Lee, C.W.; Chung, J.W. Assessment of molecular genetic diversity and population structure of sesame (Sesamum indicum L.) core collection accessions using simple sequence repeat markers. Plant. Genet. Resour. 2014, 12, 112-119. [CrossRef]

57. Adu-Gyamfi, R.; Prempeh, R.; Zakaria, I. Diversity assessment of some sesame (Sesamum indicum L.) genotypes cultivated in Northern Ghana using morphological and simple sequence repeat (SSR) markers. Adv. Agric. 2019, 1-10. [CrossRef]

58. Botstein, D.; White, R.L.; Skolnick, M.; Davis, R.W. Construction of a genetic linkage map in man using restriction fragment length polymorphism. Am. J. Hum. Genet. 1980, 32, 314-331.

59. Meszaros, K.; Karsai, I.; Kuti, C.; Banyai, J.; Lang, L.; Bedo, Z. Efficiency of different marker systems for genotype fingerprinting and genetic diversity studies in barley (Hordeum vulgare L). S. Afr. J. Bot. 2007, 73, 43-48. [CrossRef]

60. Stankiewicz, M.; Gadamski, G.; Gawronski, S.W. Genetic variation and phylogenetic relationships of triazine resistant and triazine susceptible biotypes of Solanum nigrum analysis using RAPD markers. Weed Res. 2001, 41, 287-300. [CrossRef]

61. Yol, E.; Uzun, B. Geographical patterns of sesame (Sesamum indicum L.) accessions grown under Mediterranean environmental conditions, and establishment of a core collection. Crop. Sci. 2012, 52, 2206-2214. [CrossRef]

62. Yol, E.; Toker, R.; Golukcu, M.; Uzun, B. Oil content and fatty acid characteristics in Mediterranean sesame core collection. Crop. Sci. 2015, 55, 2177-2185. [CrossRef]

63. Doyle, J.J.; Doyle, J.L. A rapid total DNA preparation procedure for fresh plant tissue. Focus 1990, $12,13-15$.

64. Girardot, C.; Scholtalbers, J.; Sauer, S.; Su, S.; Furlong, E.E.M. Je, a versatile suite to handle multiplexed NGS libraries with unique molecular identifiers. BMC Bioinform. 2016, 17, 419. [CrossRef] [PubMed]

65. Chen, S.; Zhou, Y.; Chen, Y.; Gu, J. Fastp: An ultra-fast all-in-one FASTQ preprocessor. Bioinformatics 2018, 34, 884-890. [CrossRef] 
66. Wang, L.; Xia, Q.; Zhang, Y.; Zhu, X.; Zhu, X.; Li, D.; Ni, X.; Gao, Y.; Xiang, H.; Wei, X.; et al. Updated sesame genome assembly and fine mapping of plant height and seed coat color QTLs using a new high-density genetic map. BMC Genom. 2016, 17, 31. [CrossRef] [PubMed]

67. Langmead, B.; Salzberg, S.L. Fast gapped-read alignment with bowtie 2. Nat. Methods 2012, 9, 357-359. [CrossRef] [PubMed]

68. Garrison, E.; Marth, G. Haplotype-based variant detection from short-read sequencing. arXiv 2012, arXiv:1207.3907.

69. Freese, N.H.; Norris, D.C.; Loraine, A.E. Integrated genome browser: Visual analytics platform for genomics. Bioinformatics 2016, 32, 2089-2095. [CrossRef]

70. Untergasser, A.; Nijveen, H.; Rao, X.; Bisseling, T.; Geurts, R.; Leunissen, J.A.M. Primer3Plus, an enhanced web interface to Primer3. Nucleic Acids Res. 2007, 35, 71-74. [CrossRef]

71. Peakall, R.; Smouse, P.E. GenAlEx 6.5: Genetic analysis in Excel. Population genetic software for teaching and research-an update. Bioinformatics 2012, 28, 2537-2539. [CrossRef]

72. Park, S.D.E. Trypano Tolerance in West African Cattle and the Population Genetic Effects of Selection. Ph.D. Thesis, Dublin University, Dublin, Ireland, 2001.

73. Tamura, K.; Peterson, D.; Peterson, N.; Stecher, G.; Nei, M.; Kumar, S. MEGA5: Molecular evolutionary genetics analysis using maximum likelihood, evolutionary distance, and maximum parsimony methods. Mol. Biol. Evol. 2011, 28, 2731-2739. [CrossRef]

(C) 2020 by the authors. Licensee MDPI, Basel, Switzerland. This article is an open access article distributed under the terms and conditions of the Creative Commons Attribution (CC BY) license (http://creativecommons.org/licenses/by/4.0/). 